\title{
Sodium-Glucose Cotransporter 2 Inhibitors Reduce Prandial Insulin Doses in Type 2 Diabetic Patients Treated With the Intensive Insulin Therapy
}

\author{
Mariko Hakoshima ${ }^{a}$, Hidekatsu Yanai ${ }^{a}$, b, c, Kouki Kakuta ${ }^{a}$, \\ Hiroki Adachi ${ }^{\text {a }}$
}

\begin{abstract}
Background: Sodium-glucose cotransporter 2 inhibitors (SGLT2i) are anti-diabetic drugs which improve blood glucose control by blocking reabsorption of glucose from the proximal tubule of kidney. Anti-atherosclerotic properties and cardiovascular protective effects of SGLT2i have been demonstrated by recent studies; however, the efficacy and safety of addition of SGLT2 $i$ to the intensive insulin therapy remain largely unknown.
\end{abstract}

Methods: We retrospectively picked up patients hospitalized for treatment of type 2 diabetes, who had been treated by the intensive insulin therapy and whose treatment using by SGLT2i started during their hospitalization. Such patients were picked up between June 2014 and May 2017 based on medical charts.

Results: We found 12 eligible patients. Observation period was $10.2 \pm$ 4.7 days, and SGLT2i was started at $12.2 \pm 12.9$ days after the admission. During observation period, nobody developed hypoglycemia. In spite of showing decrease of blood glucose (non-significant) before each meal, the addition of SGLT2i significantly reduced daily prandial insulin doses by approximately 4.6 units/day (-66\%). The SGLT2i addition also decreased body weight by approximately $1.3 \mathrm{~kg}$.

Conclusion: Present study demonstrated that the addition of SGLT2i to intensive insulin therapy reduced prandial insulin doses and body weight, without the development of hypoglycemia. This result may be due to SGLT2i-mediated improvement of postprandial hyperglycemia by increasing urinary glucose excretion not via insulin secretion.

Keywords: Body weight; Hemoglobin A1c; Intensive insulin thera-

Manuscript submitted February 28, 2018, accepted March 12, 2018

a Department of Internal Medicine, National Center for Global Health and Medicine Kohnodai Hospital, Chiba, Japan

${ }^{\mathrm{b}}$ Clinical Research and Trial Center, National Center for Global Health and Medicine Kohnodai Hospital, Chiba, Japan

${ }^{\mathrm{c}}$ Corresponding Author: Hidekatsu Yanai, Department of Internal Medicine, National Center for Global Health and Medicine Kohnodai Hospital, 1-7-1 Kohnodai, Ichikawa, Chiba 272-8516, Japan.

Email: dyanai@hospk.ncgm.go.jp

doi: https://doi.org/10.14740/jocmr3392w py; Sodium-glucose cotransporter 2 inhibitor; Urinary glucose excretion

\section{Introduction}

Sodium-glucose cotransporter 2 (SGLT2) is expressed in the proximal tubule of kidney and mediates reabsorption of glucose [1], and SGLT2 inhibitors (SGLT2i) prevent reabsorption of glucose by inhibiting SGLT2, therefore, SGLT2i improve glycemic control, in a dependent manner of the estimated glomerular filtration rate (eGFR) [2-4].

We previously presented the hypothesis for possible antiatherosclerotic effects of SGLT2i [5]. Briefly, caloric loss by SGLT2 inhibition may decrease plasma glucose without increasing insulin secretion, which may reduce body weight and result in improvement of insulin sensitivity. An improvement of insulin resistance may ameliorate atherosclerotic risk factors such as dyslipidemia, hypertension and elevated inflammatory cytokines [5]. Furthermore, we showed that SGLT2i improve various metabolic parameters including coronary risk factors, in the real world $[6,7]$.

The EMPA-REG OUTCOME, a randomized placebocontrolled trial (RCT) that examined the effect of empagliflozin in addition to standard of care in patients with type 2 diabetes and established cardiovascular (CV) diseases demonstrated a significant reduction in the incidence of $\mathrm{CV}$ death and heart failure hospitalization [8]. Recently, the Canagliflozin Cardiovascular Assessment Study (CANVAS) program also reported the preventing effects of canagliflozin on CV events [9]. Further, both RCTs showed renal protective effects of SGLT2i $[9,10]$, which may be associated with cardio-protective effects of SGLT2i [11]. Such renal and CV protective effects of SGLT2i were also observed in our previous studies [12-14].

Large outcomes trials of more versus less intense glucose lowering in which insulin was used in both study groups have not shown a clear CV benefit [15], and one trial showed increased mortality [16]. The risk of hypoglycemia and the suggestion that insulin might promote $\mathrm{CV}$ have raised concerns regarding the safety of insulin for type 2 diabetes $[17,18]$.

The effects of addition of SGLT2i to the intensive insulin therapy remain largely unknown. Here, we retrospectively 


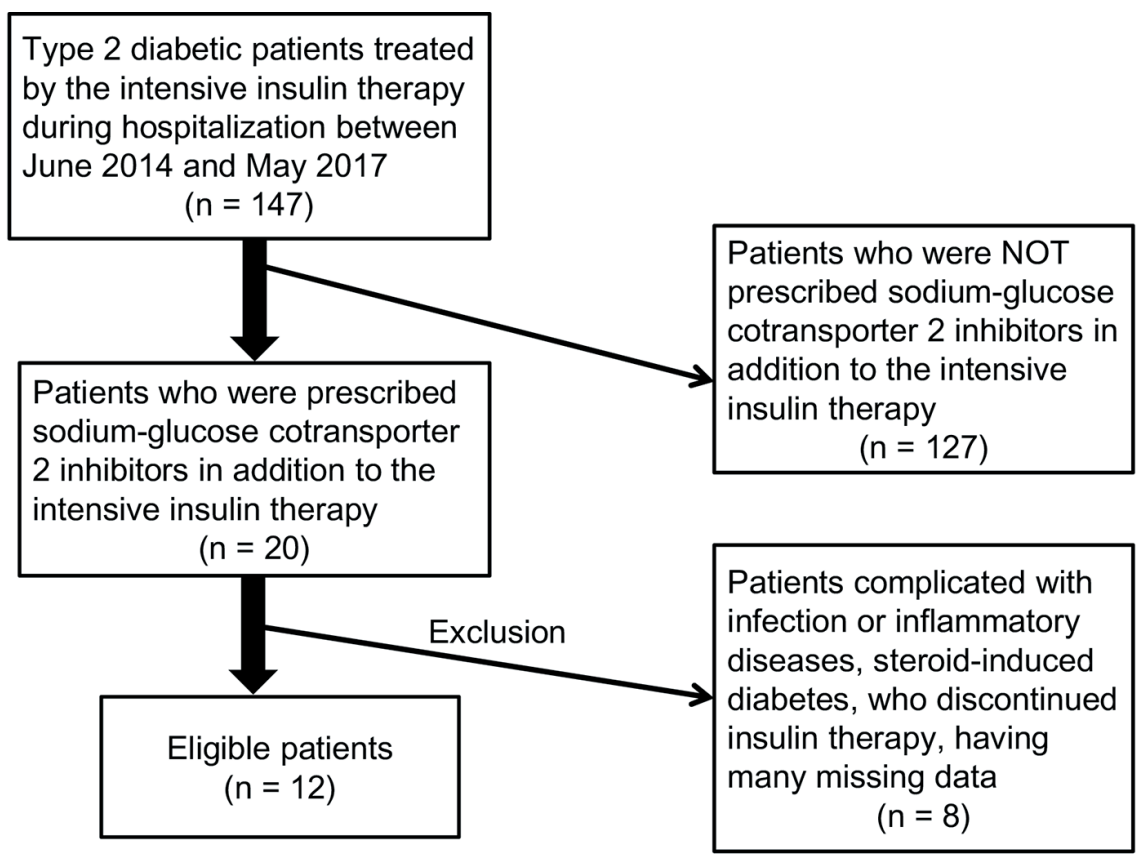

Figure 1. Recruitment of patients studied.

studied the effects of the addition of SGLT2i on blood glucose and daily prandial and basal insulin doses in type 2 diabetic hospitalized patients who had been treated with the intensive insulin therapy.

\section{Materials and Methods}

This study was approval by the Institutional Ethics Committee in National Center for Global Health and Medicine, and was also performed in accordance with the Declaration of Helsinki.

We selected patients hospitalized for treatment of type 2 diabetes, who had been treated by the intensive insulin therapy and whose treatment using by SGLT2 $\mathrm{i}$ started during their hospitalization. Such patients were picked up between June 2014 and May 2017 based on medical charts. Patients complicated with infection or inflammatory diseases, steroid-induced diabetes, and who discontinued insulin therapy, having many missing data were excluded. We compared the data before and after the start of SGLT2i. We obtained data about age, sex, body height, body weight, blood glucose levels and insulin doses before breakfast, lunch and dinner, and at bedtime, plasma glucose, hemoglobin A1c (HbA1c), serum low-density lipoprotein-cholesterol, triglyceride, high-density lipoproteincholesterol, uric acid, aspartate aminotransferase, alanine aminotransferase and $\gamma$-glutamyltransferase and creatinine measured at the baseline. Body weight, blood pressure, blood glucose levels and insulin doses before breakfast, lunch and dinner, and at bedtime, and the area under the curve (AUC) of blood glucose, and daily total insulin doses, daily total prandial insulin doses, and daily basal insulin doses, before the start of SGLT2i were compared with those after the start of SGLT2i. The period in which blood glucose levels and insulin doses before breakfast, lunch and dinner, at bedtime were described before and after administration of SGLT2i, was adopted as the observation period.

Comparison of the variables determined before and after was analyzed by a paired Student's $t$-test. All data are expressed as mean $\pm \mathrm{SD}$. $\mathrm{P}<0.05$ and $\mathrm{P}<0.1$ were considered to be statistically significant and to show tendency, respectively.

\section{Results}

Recruitment of patients studied was shown in Figure 1. We found 12 eligible patients (male/female, 4/8). Clinical and biochemical characteristics of patients studied were shown in Table 1. Prescribed anti-diabetic drugs including SGLT2i in addition to the intensive insulin therapy were shown in Table 2.

Observation period was 10.2 \pm 4.7 days, and SGLT2i was started at $12.2 \pm 12.9$ days after the admission. During observation period, nobody developed hypoglycemia.

The addition of SGLT2 $i$ to the intensive insulin therapy tended to decrease fasting blood glucose, and did not significantly change blood glucose levels before lunch, dinner and at bedtime (Fig. 2). However, the addition of SGLT2i tended to reduce insulin doses before breakfast and dinner, and also significantly reduced insulin dose before lunch (Fig. 2).

Both systolic $(123.3 \pm 17.1 \mathrm{~mm} \mathrm{Hg})$ and diastolic blood pressure $(79.8 \pm 10.4 \mathrm{~mm} \mathrm{Hg})$ did not change after the start of SGLT2i; systolic and diastolic blood pressures after the start of SGLT2i were $124.5 \pm 17.8$ and $78.6 \pm 10.1 \mathrm{~mm} \mathrm{Hg}$, respectively. The SGLT2i addition significantly reduced body weight, and did not show a significant difference in the AUC of blood glucose between before and after the start of SGLT2 $\mathrm{i}$ 
Table 1. Clinical and Biochemical Characteristics (Mean \pm SD) of Patients at Baseline

\begin{tabular}{ll}
\hline Age (years) & $56.1 \pm 13.0$ \\
Body height $(\mathrm{cm})$ & $160.4 \pm 10.0$ \\
Body weight $(\mathrm{kg})$ & $71.5 \pm 15.2$ \\
Body mass index $\left(\mathrm{kg} / \mathrm{m}^{2}\right)$ & $26.1 \pm 10.4$ \\
Systolic blood pressure (mm Hg) & $123.3 \pm 17.1$ \\
Diastolic blood pressure (mm Hg) & $79.8 \pm 10.4$ \\
HbA1c (\%) & $12.1 \pm 2.9$ \\
Aspartate aminotrasferase (U/L) & $21.8 \pm 7.1$ \\
Alanine aminotrasferase (U/L) & $25.9 \pm 11.2$ \\
$\gamma$-glutamyltransferase (U/L) & $40.6 \pm 24.8$ \\
Uric acid (mg/dL) & $5.0 \pm 1.3$ \\
Creatinine (mg/dL) & $0.7 \pm 0.1$ \\
Triglyceride (mg/dL) & $191.1 \pm 106.1$ \\
High-density lipoprotein-cholesterol (mg/dL) & $56.2 \pm 30.3$ \\
Low-density lipoprotein-cholesterol (mg/dL) & $93.0 \pm 19.6$ \\
\hline
\end{tabular}

(Fig. 3).

The SGLT2i addition did not show a significant difference in total insulin doses and daily basal insulin doses, however,
Table 2. Prescribed Anti-Diabetic Drugs in Addition to the Intensive Insulin Therapy

\begin{tabular}{ll}
\hline Anti-diabetic drugs & n \\
\hline Sodium-glucose cotransporter 2 inhibitors & 12 \\
Dapagliflozin & 6 \\
Luseogliflozin & 6 \\
Metformin & 9 \\
Pioglitazone & 3 \\
Dipeptidyl peptidase-4 inhibitors & 6 \\
Glucagon-like peptide-1 analogues & 5 \\
a-glucosidase inhibitors & 3 \\
\hline
\end{tabular}

significantly reduced daily prandial insulin doses (Fig. 4).

\section{Discussion}

Anti-atherosclerotic properties and $\mathrm{CV}$ protective effects of SGLT2i have been demonstrated by recent RCTs and observational studies [6-9, 19]. Regarding the insulin therapy for patients with type 2 diabetes, controversial discussions exist. The Action to Control Cardiovascular Risk in Diabetes (AC-
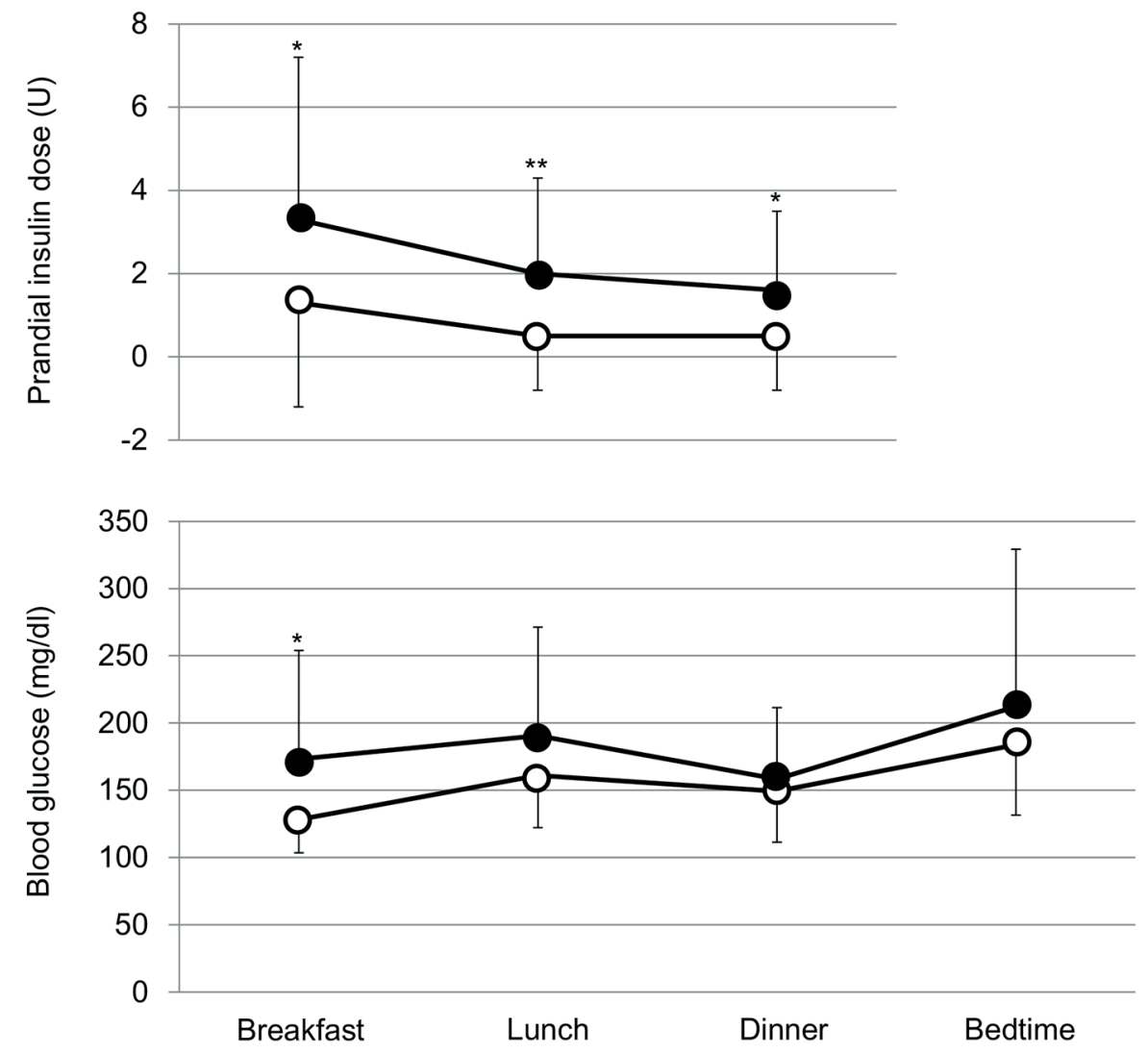

Figure 2. Comparison of prandial insulin doses and blood glucose levels before and after the addition of SGLT2i. Black and white circles indicate values before and after the start of SGLT2i, respectively. ${ }^{*} \mathrm{P}<0.1$ and ${ }^{* *} \mathrm{P}<0.05$ vs. after the start of $S G L T 2 i$. 

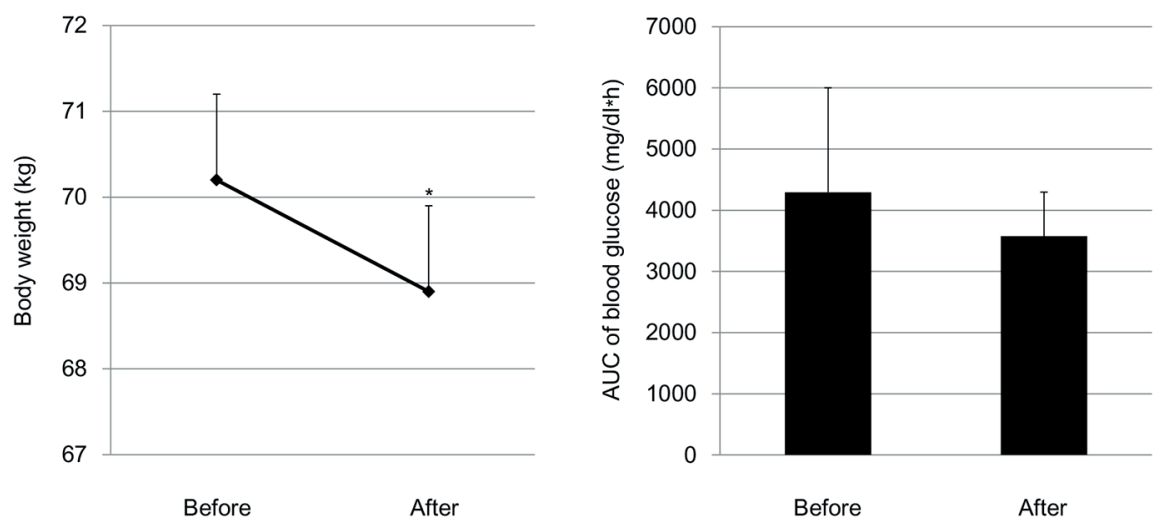

Figure 3. Changes in body weight and area under the curve (AUC) of blood glucose levels by the addition of SGLT2i. ${ }^{*} P<0.05$.

CORD) study showed that the use of intensive therapy to target normal glycated hemoglobin levels for 3.5 years increased mortality and did not significantly reduce major CV events as compared with standard therapy in patients with type 2 diabe-
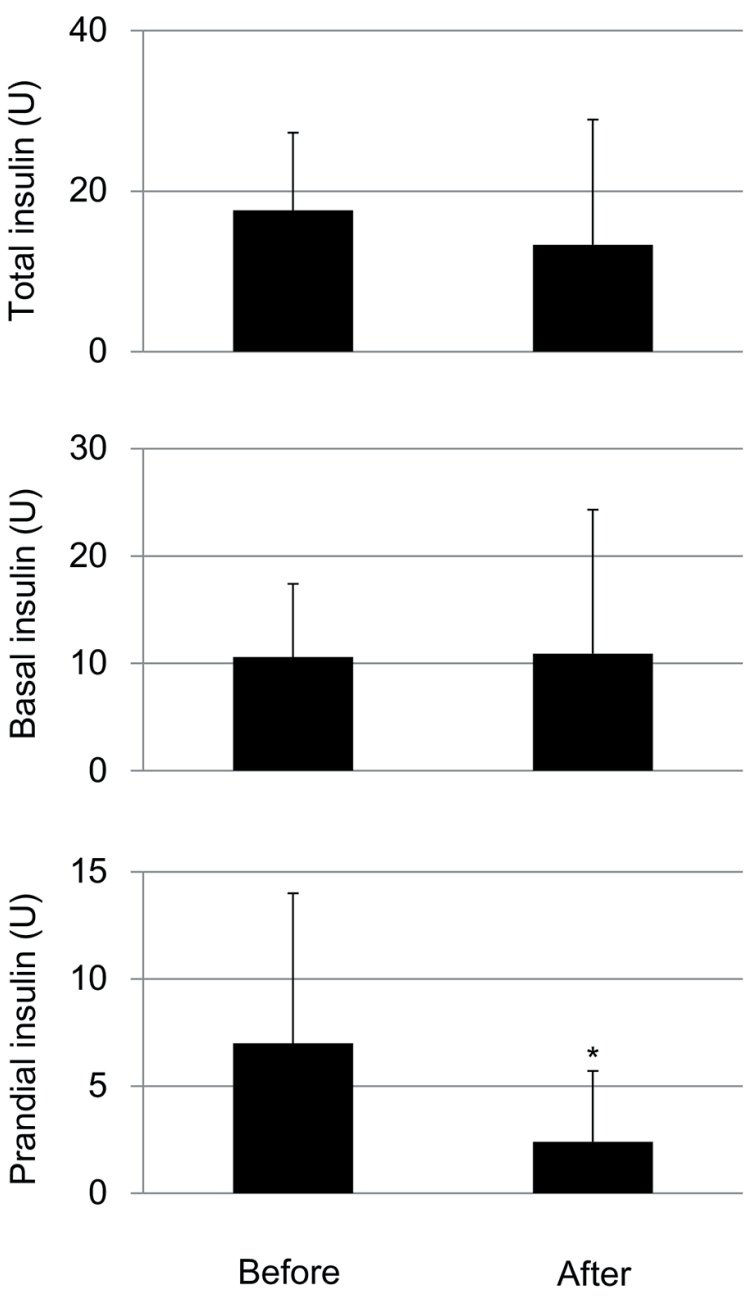

Figure 4. Changes in daily total insulin doses, basal insulin doses and daily total prandial insulin doses by the addition of SGLT2i. * $P<0.05$. tes [16]. The efficacy and safety of addition of SGLT2i to the intensive insulin therapy remain largely unknown.

Neal et al studied the efficacy and safety of canagliflozin in patients with type 2 diabetes using insulin [20]. In their study protocol, participants were required to be receiving stable background glucose-lowering therapy, including insulin.

Canagliflozin added to insulin therapy improved glycemic control and decreased body weight; however, a greater incidence of hypoglycemia was observed [20]. Rosenstock et al also reported the efficacy and safety of empagliflozin, added to multiple daily injections of insulin in obese patients with type 2 diabetes [21]. In their protocol, insulin dose was to remain stable in weeks $1-18$, adjusted to meet glucose targets in weeks 19 - 40, then stable in weeks 41 - 52. Empagliflozin improved glycemic control and reduced insulin doses ( -9 to -11 $\mathrm{U})$ without increasing the risk of hypoglycemia [21]. Wilding et al studied the efficacy and safety of adding dapagliflozin therapy in patients whose type 2 diabetes is inadequately controlled with insulin [22]. In this study, insulin doses were not titrated to target. Daily insulin dose decreased by 0.63 to $1.95 \mathrm{U}$ with dapagliflozin and increased by $5.65 \mathrm{U}$ with placebo. Compared with the placebo group, patients in the dapagliflozin groups had a higher rate of hypoglycemic episodes (56.6\% vs. 51.8\%) [22].

It has not been reported how the SGLT2i addition to intensive insulin therapy influences on prandial and basal insulin doses. Present study demonstrated that SGLT2i reduced prandial (bolus) insulin doses under the titration during hospitalization. In our study, the SGLT2i addition showed reduction of blood glucose (non-significant) before each meal, however, showed a reduction $(\mathrm{P}<0.05$ and $\mathrm{P}<0.1)$ of insulin doses before breakfast, lunch and dinner, which suggests an improvement of postprandial hyperglycemia by SGLT2i. Kakuda et al reported that tofogliflozin (SGLT2i) treatment causes an improvement of postprandial glucose metabolism [23]. Nishimura et al also showed that SGLT2i reduced postprandial hyperglycemia by increasing postprandial urinary glucose excretion, by using continuous glucose monitoring system [24]. These studies support our hypothesis.

In the ACCORD study, hypoglycemia requiring assistance and weight gain of more than $10 \mathrm{~kg}$ were more frequent in the intensive therapy group $(\mathrm{P}<0.001)$ [16]. In present study, the addition of SGLT2i to the intensive insulin therapy reduced 
both insulin doses and body weight without the development of severe hypoglycemia, which suggests this combination is beneficial for $\mathrm{CV}$ events.

The present study has several limitations. First, other hypoglycemic, anti-hypertensive, or lipid lowering agents, food intakes and/or exercise levels may have an influence on the study results. Second, the number of studied subjects was small because of the limited availability. A more detailed prospective study is recommended to evaluate the effects of SGLT2 $\mathrm{i}$ in addition to the intensive insulin therapy more validly.

The present study also has the strength. First, metabolic parameters were measured by the same laboratory. Second, subjects were treated by the specialists for diabetes treatment who were equally educated by the same specialist. Third, to our knowledge, this study is the first to show precise effects of the addition of SGLT2i to the intensive insulin therapy (three times bolus and basal insulin injections) on daily changes of blood glucose and insulin doses in patients with type 2 diabetes, under the condition of hospitalization.

\section{Conclusion}

Our study demonstrated that the addition of SGLT2i to intensive insulin therapy reduced prandial insulin doses and body weight, without the development of hypoglycemia. This result may be due to SGLT2i-mediated improvement of postprandial hyperglycemia by increasing urinary glucose excretion not via insulin secretion.

\section{Acknowledgments}

We thank Tomoko Kaga and other staffs of Clinical Research and Trial Center, National Center for Global Health and Medicine Kohnodai Hospital, for valuable technical help.

\section{Author Contributions}

M.H. and H.Y. designed the research. M.H., H.A., and K.K. collected data. H.Y. analyzed data and wrote the paper. All authors read and approved the final paper.

\section{Conflict of Interest}

The authors declare that they have no conflict of interest concerning this article.

\section{References}

1. Vallon V, Platt KA, Cunard R, Schroth J, Whaley J, Thomson SC, Koepsell H, et al. SGLT2 mediates glucose reabsorption in the early proximal tubule. J Am Soc Nephrol. 2011;22(1):104-112.

2. Jabbour SA, Goldstein BJ. Sodium glucose co-transport- er 2 inhibitors: blocking renal tubular reabsorption of glucose to improve glycaemic control in patients with diabetes. Int J Clin Pract. 2008;62(8):1279-1284.

3. Katsuyama H, Yanai H. An influence of the estimated glomerular filtration rate on improvement in metabolic parameters by sodium-glucose cotransporter 2 inhibitors. J Clin Med Res. 2016;8(6):486-488.

4. Yanai H, Hakoshima M, Adachi H. What Properties of Sodium-Glucose Cotransporter 2 Inhibitors Determine the Improvement in Hemoglobin A1c and Body Weight? J Clin Med Res. 2017;9(5):446-448.

5. Yanai H, Katsuyama H, Hamasaki H, Adachi H, Moriyama S, Yoshikawa R, Sako A. Sodium-glucose cotransporter 2 inhibitors: possible anti-atherosclerotic effects beyond glucose lowering. J Clin Med Res. 2016;8(1):1014.

6. Katsuyama H, Hamasaki H, Adachi H, Moriyama S, Kawaguchi A, Sako A, Mishima S, et al. Effects of sodium-glucose cotransporter 2 inhibitors on metabolic parameters in patients with type 2 diabetes: a chart-based analysis. J Clin Med Res. 2016;8(3):237-243.

7. Yanai H, Hakoshima M, Adachi H, Kawaguchi A, Waragai Y, Harigae T, Masui Y, et al. Effects of six kinds of sodium-glucose cotransporter 2 inhibitors on metabolic parameters, and summarized effect and its correlations with baseline data. J Clin Med Res. 2017;9(7):605-612.

8. Zinman B, Wanner C, Lachin JM, Fitchett D, Bluhmki E, Hantel S, Mattheus M, et al. Empagliflozin, cardiovascular outcomes, and mortality in type 2 diabetes. N Engl J Med. 2015;373(22):2117-2128.

9. Neal B, Perkovic V, Mahaffey KW, de Zeeuw D, Fulcher G, Erondu N, Shaw W, et al. Canagliflozin and cardiovascular and renal events in type 2 diabetes. $\mathrm{N}$ Engl J Med. 2017;377(7):644-657.

10. Wanner C, Inzucchi SE, Lachin JM, Fitchett D, von Eynatten M, Mattheus M, Johansen OE, et al. Empagliflozin and progression of kidney disease in type 2 diabetes. N Engl J Med. 2016;375(4):323-334.

11. Yanai H. Sodium-glucose cotransporter 2 inhibitors and death and heart failure in type 2 diabetes. Ann Transl Med. 2017;5(23):470.

12. Yanai H, Katsuyayama H. A Possible Mechanism for Renoprotective Effect of sodium-glucose cotransporter 2 inhibitor: elevation of erythropoietin production. J Clin Med Res. 2017;9(2):178-179.

13. Koguchi A, Adachi H, Yanai H. The application of sodium-glucose cotransporter 2 inhibitors to chronic kidney disease stage 4. J Clin Med Res. 2017;9(12):1029-1031.

14. Yanai H, Adachi H, Hakoshima M. Understanding of hypertension and heart failure in patients with type 2 diabetes by studying effects of sodium-glucose cotransporter 2 inhibitors on plasma B-type natriuretic peptide levels. J Clin Hypertens (Greenwich). 2018;20(2):411-412.

15. Control G, Turnbull FM, Abraira C, Anderson RJ, Byington RP, Chalmers JP, Duckworth WC, et al. Intensive glucose control and macrovascular outcomes in type 2 diabetes. Diabetologia. 2009;52(11):2288-2298.

16. Action to Control Cardiovascular Risk in Diabetes Study G, Gerstein HC, Miller ME, Byington RP, Goff 
DC, Jr., Bigger JT, Buse JB, et al. Effects of intensive glucose lowering in type 2 diabetes. N Engl J Med. 2008;358(24):2545-2559.

17. Yakubovich N, Gerstein HC. Serious cardiovascular outcomes in diabetes: the role of hypoglycemia. Circulation. 2011;123(3):342-348.

18. Mellbin LG, Malmberg K, Norhammar A, Wedel H, Ryden L, Investigators D. Prognostic implications of glucose-lowering treatment in patients with acute myocardial infarction and diabetes: experiences from an extended follow-up of the Diabetes Mellitus Insulin-Glucose Infusion in Acute Myocardial Infarction (DIGAMI) 2 Study. Diabetologia. 2011;54(6):1308-1317.

19. Kosiborod M, Cavender MA, Fu AZ, Wilding JP, Khunti K, Holl RW, Norhammar A, et al. Lower risk of heart failure and death in patients initiated on sodium-glucose cotransporter-2 inhibitors versus other glucose-lowering drugs: the CVD-REAL study (comparative effectiveness of cardiovascular outcomes in new users of sodium-glucose cotransporter-2 inhibitors). Circulation. 2017;136(3):249-259.

20. Neal B, Perkovic V, de Zeeuw D, Mahaffey KW, Fulcher G, Ways K, Desai M, et al. Efficacy and safety of canagliflozin, an inhibitor of sodium-glucose cotransporter 2, when used in conjunction with insulin therapy in patients with type 2 diabetes. Diabetes Care. 2015;38(3):403-411.

21. Rosenstock J, Jelaska A, Frappin G, Salsali A, Kim G, Woerle HJ, Broedl UC, et al. Improved glucose control with weight loss, lower insulin doses, and no increased hypoglycemia with empagliflozin added to titrated multiple daily injections of insulin in obese inadequately controlled type 2 diabetes. Diabetes Care. 2014;37(7):18151823.

22. Wilding JP, Woo V, Soler NG, Pahor A, Sugg J, Rohwedder K, Parikh S, et al. Long-term efficacy of dapagliflozin in patients with type 2 diabetes mellitus receiving high doses of insulin: a randomized trial. Ann Intern Med. 2012;156(6):405-415.

23. Kakuda H, Kobayashi J, Sakurai M, Kakuda M, Takekoshi N. The effect of tofogliflozin treatment on postprandial glucose and lipid metabolism in Japanese men with type 2 diabetes: A Pilot Study. J Clin Med Res. 2017;9(5):403409.

24. Nishimura R, Omiya H, Sugio K, Ubukata M, Sakai S, Samukawa Y. Sodium-glucose cotransporter 2 inhibitor luseogliflozin improves glycaemic control, assessed by continuous glucose monitoring, even on a low-carbohydrate diet. Diabetes Obes Metab. 2016;18(7):702-706. 